\title{
Role of osteoprotegerin in the regulation of dental epithelial-mesenchymal signaling during tooth development
}

\author{
XIN GAO $^{1}$, JUNMING ZHENG ${ }^{2}$, SHAOQIN TU $^{1}$, BIN CAI $^{1}$, RONGSHENG ZENG ${ }^{1}$ and LUSAI XIANG ${ }^{1}$ \\ ${ }^{1}$ Guanghua School of Stomatology, Hospital of Stomatology, Guangdong Provincial Key Laboratory of Stomatology, \\ Sun Yat-sen University, Guangzhou, Guangdong 510055; ${ }^{2}$ Research Management Office, Foshan Stomatology Hospital, \\ School of Stomatology and Medicine, Foshan University, Foshan, Guangdong 528000, P.R. China
}

Received February 12, 2019; Accepted April 24, 2019

DOI: $10.3892 / \mathrm{mmr} .2019 .10567$

\begin{abstract}
Dental epithelial-mesenchymal signaling is crucial for tooth development, but the detailed mechanism is not fully understood. Using microarray analysis, it was revealed that the expression of osteoprotegerin, an important factor regulating bone remodeling, significantly increased after removal of the dental epithelium. Immunohistochemical staining revealed that osteoprotegerin expression within the dental mesenchyme was quite low during the prenatal period, but significantly increased after birth. To investigate the influence of osteoprotegerin upon tooth development, first-molar tooth germs from embryonic day 14.5 (E14.5) Chinese Kunming mice were treated with different concentrations of osteoprotegerin. It was revealed that osteoprotegerin could inhibit the expression of odontogenic markers while promoting the expression of osteogenic markers, thereby disrupting tooth morphogenesis. These findings were further supported by in vitro and in vivo cultures. Finally, quantitative reverse transcription-polymerase chain reaction and immunofluorescence studies revealed that, after osteoprotegerin treatment, the activity of the wingless/integrated (Wnt)/ $\beta$-catenin pathway increased, indicating that increased osteoprotegerin expression in prenatal tooth development could lead to uncontrolled upregulation of the $\mathrm{Wnt} / \beta$-catenin pathway.
\end{abstract}

Correspondence to: Dr Rongsheng Zeng or Dr Lusai Xiang, Guanghua School of Stomatology, Hospital of Stomatology, Guangdong Provincial Key Laboratory of Stomatology, Sun Yat-sen University, 56 Lingyuanxi Road, Guangzhou, Guangdong 510055, P.R. China

E-mail: gaox0724@163.com

E-mail: xiangls@mail.sysu.edu.cn

Abbreviations: RUNX2, runt-related transcription factor 2; DSPP, dentin sialophosphoprotein; DSP, dentin sialoprotein; RANKL, receptor activator of nuclear factor kappa B ligand; IHC, immunohistochemical; RT-qPCR, quantitative reverse transcription-polymerase chain reaction

Key words: osteoprotegerin, Wnt signaling pathway, odontogenesis, osteogenesis, dental epithelium, mesenchyme

\section{Introduction}

Teeth develop as a result of sequential and reciprocal interactions between the oral ectoderm and neural crest-derived mesenchyme (1). In the absence of the dental epithelium or dental mesenchyme, tooth morphogenesis would be abolished (2). Bone morphogenetic protein (3), fibroblast growth factor (4), Hedgehog (5) and wingless/integrated (Wnt) families (6) are involved in this epithelial-mesenchymal interaction.

Osteoprotegerin is a member of the tumor necrosis factor receptor superfamily (7). Lacking transmembrane and cytoplasmic domains, it is presented mainly in soluble form in extracellular fluids. Functioning as a decoy receptor for receptor activator of nuclear factor kappa B ligand (RANKL), it is an integral part of the RANKL/RANK/osteoprotegerin system because it 'tunes' the balance between the formation and resorption of bone (8). Recent studies have demonstrated that osteoprotegerin is involved in orthodontic tooth movement (9), periodontitis (10), tooth eruption (11) and various other physiologic or pathologic processes (12). However, whether osteoprotegerin is involved in tooth development has not been studied.

In the present study, osteoprotegerin expression was dynamically assessed during teeth development. In vitro and in vivo experiments were carried out to ascertain if removal of the dental epithelium could lift suppression of osteoprotegerin expression and generate an effect similar to that elicited by osteoprotegerin treatment. Finally, it was investigated whether osteoprotegerin could influence mesenchymal Wnt/ $\beta$-catenin activity (13).

\section{Materials and methods}

Ethical approval of the study protocol. The study protocol for animal studies was approved by the Ethics Committee of the Hospital of Stomatology within Sun Yat-sen University (ERC-2013-15; Guangzhou, China).

Animals. The 'tooth germ' is an aggregation of cells that eventually forms a tooth. Our aim was to isolate specimens of tooth germs at different stages of tooth development for further study, and to carry out experiments in vitro and in vivo.

Female Chinese Kunming mice ( $\mathrm{n}=9,4$ weeks old), Chinese Kunming mice ( $\mathrm{n}=20,7$ days old) and 4 male nude mice ( $n=4,4$ weeks old) were purchased from San Yet-sun 
University. For all experiments involving isolation of tooth germs, three mice were used for each group. In each experiment, the mice from all groups originated from same pregnant mouse to minimize genetic differences. In total, 6 mice were sacrifice at days E14.5 (25 $\pm 1.5 \mathrm{~g}), 3$ mice were sacrifice at days E16.5 $(25 \pm 1.5 \mathrm{~g}), 20$ mice were sacrificed at P7 $(6.4 \mathrm{~g})$ and 4 nude mice $(20 \mathrm{~g})$ were used for Subrenal capsule assays. All mice were kept at $26-28^{\circ} \mathrm{C}, 40 \%$ humidity, and 10 -h light and 14-h dark cycle. All mice were fed ad libitum with drinking water filtered and autoclaved before use.

Organ cultures. In order to study the influence of various factors on tooth-germ development in a controlled environment, tooth germs of first molars were dissected from the mandibles of embryonic day 14.5 (E14.5) mice and cultured in vitro. The tissue surrounding tooth germs was removed carefully under microscope guidance. The effect of the removal of the dental epithelium on tooth development was analyzed. First, isolated tooth germs were incubated in $1.2 \mathrm{U} / \mathrm{ml}$ of dispase II (cat. no. 17105041; Gibco; Thermo Fisher Scientific, Inc.) for $5 \mathrm{~min}$ at room temperature, and then the dental mesenchyme was isolated with a fine needle. Tooth germs and the isolated dental mesenchyme were cultured for 7 days on 6 -well Transwell $^{\mathrm{TM}}$ plates with 1,000 $\mu \mathrm{l} /$ well of Dulbecco's modified Eagle's medium (cat. no. 11885092; Gibco; Thermo Fisher Scientific, Inc.) supplemented with $10 \%$ fetal bovine serum, $100 \mu \mathrm{g} / \mathrm{ml}$ of ascorbic acid (cat. no. PHR1008; Sigma-Aldrich; Merck KGaA) and $2 \mathrm{mM}$ of L-glutamine (cat. no. 25030081, Gibco; Thermo Fisher Scientific, Inc.). Recombinant mouse osteoprotegerin protein $(100$ or $200 \mathrm{ng} / \mathrm{ml}$; cat. no. 375-TL; R\&D Systems) was added to the culture medium to study its effect on tooth development. Cultures were incubated at $37^{\circ} \mathrm{C}$ in a humidified atmosphere of $5 \% \mathrm{CO}_{2}$. The culture medium was changed every 3 days.

Tissue preparation and histology. To assess osteoprotegerin expression dynamically during tooth development, mice were sacrificed at E14.5, E16.5, post-partum day 1 (P1), P3, P5 and P7. Embryos at E14.5 and E16.5 and mandibles from P1, P3, $\mathrm{P} 5$, and $\mathrm{P} 7$ were fixed with $4 \%$ paraformaldehyde for $72 \mathrm{~h}$ at room temperature. Then, the tooth germs from first molars were dissected. Following decalcification with $0.5 \mathrm{M}$ of ethylenediamine tetraacetic acid solution for 2 weeks, samples were dehydrated with graded solutions of alcohol and embedded. Tooth germs from E14.5 mice were dehydrated with graded solutions of alcohol and embedded without decalcification.

Expression of osteoprotegerin, the osteogenic marker osteocalcin, and the odontogenic marker dentin sialoprotein (DSP) was assessed by immunohistochemical (IHC) staining. Anti-osteoprotegerin polyclonal antibody $(5 \mu \mathrm{g} / \mathrm{ml}$; cat no. AF459; R\&D Systems), anti-osteocalcin polyclonal antibody (50 $\mu \mathrm{l} / \mathrm{ml}$; cat. no. ab93876; Abcam) and anti-DSP monoclonal antibody ( $2 \mu \mathrm{g} / \mathrm{ml}$; cat. no. MABT37; EMD Millipore) were used as primary antibodies. Terminal deoxynucleotidyl transferase dUTP nick end labeling (TUNEL; cat. no. ab206386; Abcam) staining was carried out according to manufacturer's instructions to evaluate apoptosis activity in tooth germs with/without osteoprotegerin treatment.

Immunofluorescence analyses were carried out to evaluate the nuclear translocation of $\beta$-catenin. Tissue sections were incubated with anti- $\beta$-catenin polyclonal antibody $(50 \mu \mathrm{l} / \mathrm{ml}$; cat. no. ab2365; Abcam) overnight at $4^{\circ} \mathrm{C}$. Then, the sections were incubated with secondary antibody Alexa Fluor 488 (1:1,000 dilution; cat. no. A-21206; Invitrogen; Thermo Fisher Scientific, Inc.) for $1 \mathrm{~h}$ in a dark chamber. Finally, tissue sections were counterstained with 4'6-diamidino-2-phenylindole (DAPI; $0.5 \mu \mathrm{g} / \mathrm{ml}$ ) for $15 \mathrm{~min}$ for nuclear labeling.

Quantitative reverse transcription-polymerase chain reaction $(R T-q P C R)$. Total RNA was extracted from tooth germs or samples of isolated dental mesenchyme using PureLink ${ }^{\circledR}$ RNA Mini Kit (cat. no. 12183018A; Invitrogen; Thermo Fisher Scientific, Inc.) according to manufacturer's instructions. Complimentary-DNA synthesis was performed with random 6-mer primers using a PrimeScript ${ }^{\mathrm{TM}}$ 1st Strand cDNA Synthesis kit (cat. no.6110A; Takara Bio, Inc.). Messenger-RNA expression was assessed by RT-qPCR using the SYBR ${ }^{\circledR}$-Green method. The thermocycling conditions were: Initial denaturation for $30 \mathrm{sec}$ at $95^{\circ} \mathrm{C}$, followed by 40 cycles of $5 \mathrm{sec}$ at $95^{\circ} \mathrm{C}$ and $30 \mathrm{sec}$ at $60^{\circ} \mathrm{C}$. The relative fold change for expression of the target gene was calculated following the method proposed by of Livak and Schmittgen (14). Glyceraldehyde-3-phosphate dehydrogenase was used as endogenous reference for normalization, and the expression level in tooth germ with no osteoprotegerin treatment as a calibrator. The primers employed are listed in Table SI.

Subrenal capsule assays. Subrenal capsule assays were carried out to confirm the findings from in vitro experiments and compare tooth development between whole tooth germs and dental-mesenchyme tissue over longer periods of time. Using gel sponges as scaffolds, tooth germs or dental-mesenchyme tissue were transplanted under the subrenal capsule of 4-week-old nude mice according to the protocol of Fingert et al (15). After 3 weeks, the mice were sacrificed to obtain the transplanted tissue for further study.

Protein microarrays. In order to compare the expression of various extracellular cytokines between tooth germs and those in isolated dental-mesenchyme tissue (Fig. S1), the changes introduced by removal of the dental epithelium using protein microarray assays were identified. Quantibody ${ }^{\circledR}$ glass-based antibody arrays (product code: QAM-CAA-4000; RayBiotech) were used to assess cytokine levels. First, the glass chips were blocked with $100 \mu 1$ of diluted samples and incubated on shakers for $1 \mathrm{~h}$. After decanting buffer from each well, $100 \mu \mathrm{l}$ of samples were added, and incubated overnight at $4^{\circ} \mathrm{C}$. Microarray assays and data analyses were carried out according to manufacturer's protocols. A stock culture medium without growth factors was used as a negative control. In each chip, there were two positive controls providing a reference for normalization among different chips. The fold change in protein level was calculated using the following equation:

$$
\text { Fold change }=\log _{2} \frac{V_{\text {tooth germ }}}{V_{\text {mesenchyme }}}
$$

where $V_{\text {tooth germ }}$ denotes the microarray reading for expression of a certain protein from tooth-germ culture fluid, and $V_{\text {mesenchyme }}$ is the reading for that protein from isolated dental-mesenchyme culture fluid. 

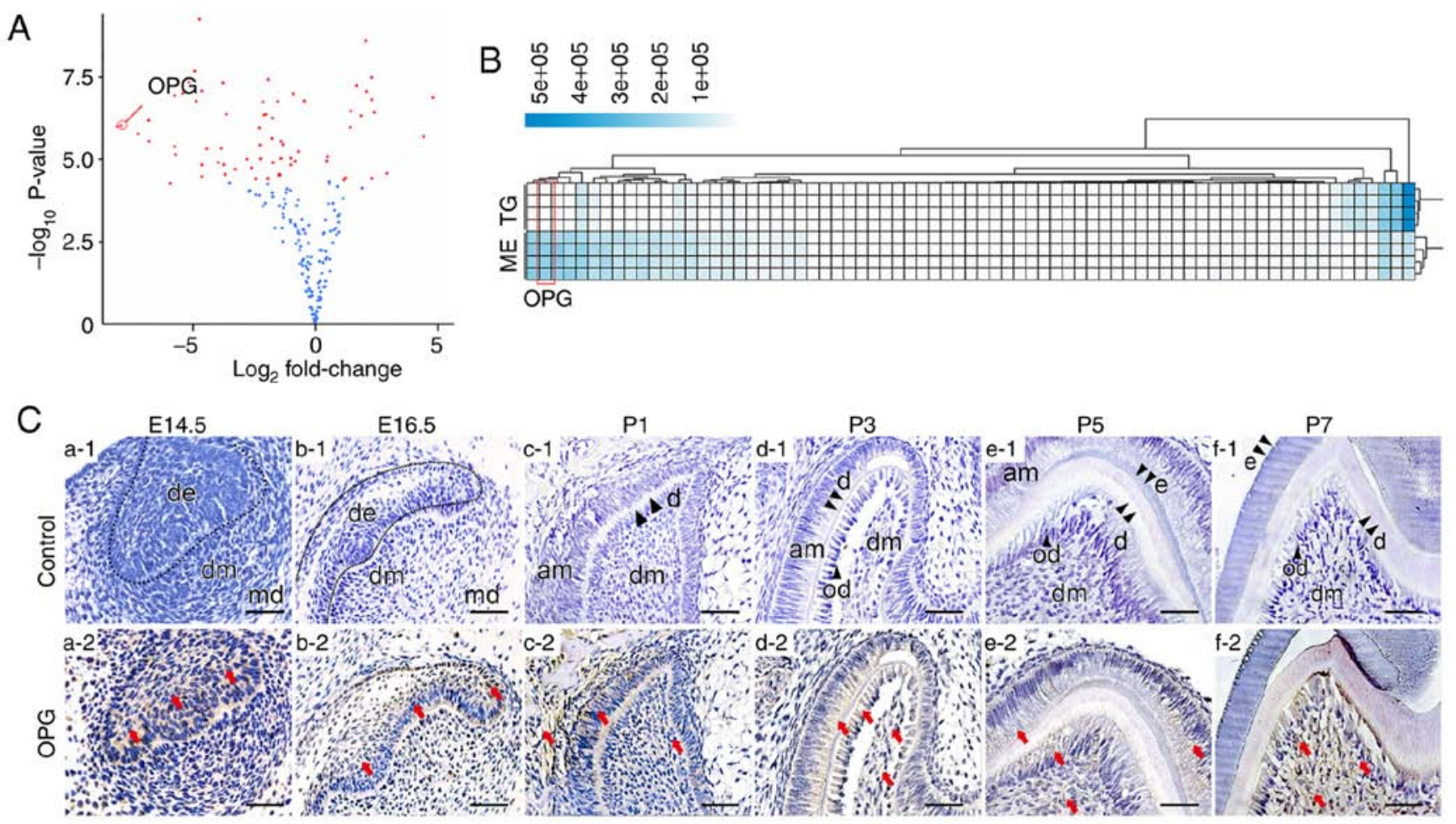

Figure 1. Expression pattern of OPG. (A and B) Volcano plot and heatmap of differential protein expression comparing extracellular protein levels between

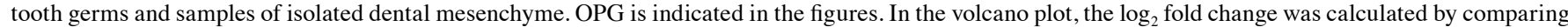
tooth germs vs. the dental mesenchyme. Microarray analysis for tooth germs and the dental mesenchyme was performed in triplicate. (C) Spatial-temporal pattern of OPG expression during tooth development. At first, OPG expression was confined to the dental epithelium (a1 and a2). Then, OPG expression in the dental mesenchyme gradually increased as development proceeded (b-f). (C) The red arrows indicate parts of the tissue that were positive for immunohistochemical staining. Scale bar, $50 \mu \mathrm{m}$. OPG, osteoprotegerin; TG, tooth germ; ME, dental mesenchyme; am, ameloblasts; d, dentin; de, dental epithelium; $\mathrm{dm}$, dental mesenchyme; md, mandible; od, odontoblast.

Statistical analysis. Upon confirmation of a normal distribution of data, all quantitative datasets were subjected to Student's t-tests or one-way analysis of variance (ANOVA), with $\mathrm{P}<0.05$ considered to indicate a statistically significant difference. Dunnett's test was used for post hoc test following ANOVA. Statistical analyses were carried out using R 3.4.2 (R Foundation). Heatmaps for differential expression of proteins were created with pheatmap (R Foundation). All experiments were performed in triplicate.

\section{Results}

Influence of the dental epithelium on extracellular levels of cytokines secreted from tooth germs. Results from protein microarray analysis revealed that removal of the dental epithelium altered expression of various secreted proteins, among which changes in osteoprotegerin expression was one of the most significant $(\mathrm{P}<0.001$; Fig. 1A). Heatmaps of microarray data further demonstrated a consistent difference in osteoprotegerin expression between culture fluid from the dental mesenchyme and whole tooth germs (Fig. 1B).

Osteoprotegerin expression at different stages of tooth development was evaluated further by IHC staining. At E14.5, osteoprotegerin expression was found only in the epithelium of internal and external enamel (Fig. 1C-a1 and a2), and a small amount of osteoprotegerin was found in the dental mesenchyme at E16.5 (Fig. 1C-b1 and b2). As development proceeded, osteoprotegerin expression in the dental mesenchyme became more prominent, but its level in the epithelium did not change much until P3. As the dental epithelium differentiated into ameloblasts, osteoprotegerin expression in the dental epithelium also decreased (Fig. 1C-c-f).

Influence of osteoprotegerin on development of tooth germs. To study the influence of osteoprotegerin on the development of tooth germs, extraneous osteoprotegerin protein (100 or $200 \mathrm{ng} / \mathrm{ml}$ ) was added to the culture medium of the first-molar tooth germs from E14.5. Tooth-germ structures in the control group (Fig. 2A-b1) and the osteoprotegerin $(100 \mathrm{ng} / \mathrm{ml})$ group (Fig. 2A-b2) were normal. However, considerable structural disruption was revealed in the osteoprotegerin $(200 \mathrm{ng} / \mathrm{ml})$ group (Fig. 2A-b3), whereby the polarity and continuity of tooth structures were lost. Staining with Masson's trichrome also revealed disrupted dentin formation in the osteoprotegerin $(200 \mathrm{ng} / \mathrm{ml})$ group (Fig. 2A-c). TUNEL staining revealed no significant difference in number of positive staining cells between the osteoprotegerin-treated groups and the control group (Fig. 2B). These results demonstrated that, during the early stages of tooth development, a high concentration of osteoprotegerin disrupted the developmental process through mechanisms other than cytotoxicity.

The influence of osteoprotegerin on various osteogenic markers was evaluated by RT-qPCR. Compared with the control group, expression of osteocalcin ( $\mathrm{P}<0.001$; Fig. 2D-b), osteopontin ( $\mathrm{P}<0.001$; Fig. 2D-c) and runt-related transcription factor 2 (RUNX2) $(\mathrm{P}<0.001$; Fig. 2D-d) was higher in the 
A
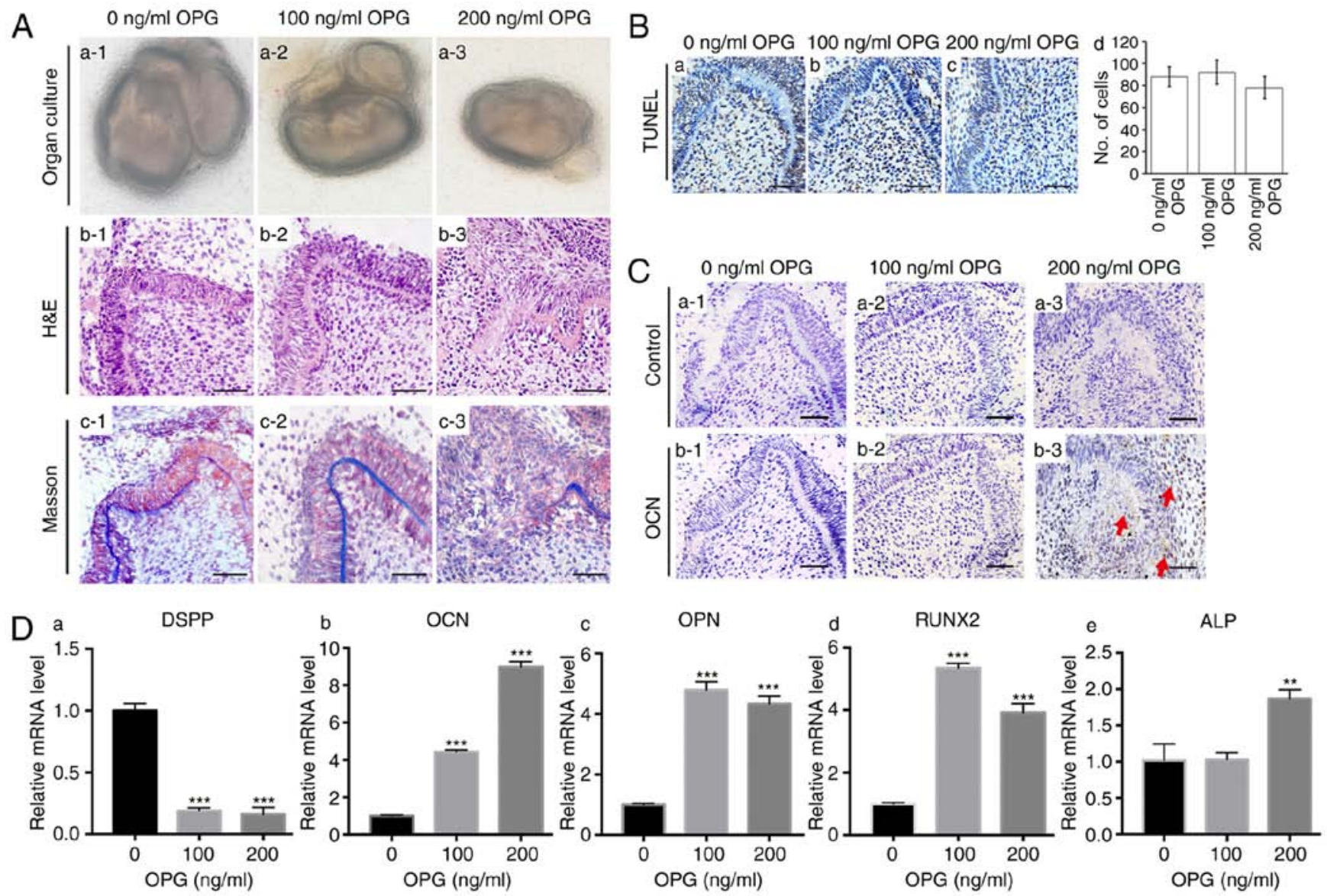

Figure 2. Effects of OPG on tooth development. (A) (a1-a3) Tooth germs of first molars were isolated from E14.5 mandibles. (b1-b3) H\&E staining of tissue sections demonstrated tooth-structure disruption after stimulation with $200 \mathrm{ng} / \mathrm{ml}$ of OPG. (c1-c3) Staining (Masson's trichrome) of collagen structures demonstrated disruption of dentin structure after stimulation with $200 \mathrm{ng} / \mathrm{ml}$ of OPG. (B) (a-c) TUNEL staining of tissue sections revealed no significant cytotoxic effect after OPG stimulation. (d) Quantification of positive cells for TUNEL staining. (C) Immunohistochemical staining of OPG in tooth germs treated with different concentrations of OPG. OCN expression was not high enough to be detected in the control group (b1) or $100 \mathrm{ng} / \mathrm{ml}$ OPG-treated group (b2), however, $200 \mathrm{ng} / \mathrm{ml}$ of OPG significantly increased OCN levels in the dental mesenchyme (b3). Three tooth germs of E14.5 mice first molars were used in each group. (D) RT-qPCR revealed that expression of the odontogenic marker DSPP (a) was suppressed after OPG stimulation. OPG increased levels of the osteogenic markers OCN (b), OPN (c), RUNX2 (d) and ALP (e). Each experiment was performed in triplicate. Scale bar, $50 \mu \mathrm{m}$ (A-b1-b3, A-c1-c3, B and C). Data are presented as the mean \pm SD. ${ }^{* *} \mathrm{P}<0.01$ and ${ }^{* * * *} \mathrm{P}<0.001$. The red arrows indicate parts of the tissue that were positive for immunohistochemical staining. OPG, osteoprotegerin; OCN, osteocalcin; DSPP, dentin sialophosphoprotein; OPN, osteopontin; RUNX2, runt-related transcription factor 2; ALP, alkaline phosphatase.

osteoprotegerin-treated groups, whereas an increase in alkaline phosphatase (ALP) was revealed only in the osteoprotegerin $(200 \mathrm{ng} / \mathrm{ml})$ group $(\mathrm{P}<0.01$; Fig. 2D-e). IHC staining further confirmed that treatment with osteoprotegerin $(200 \mathrm{ng} / \mathrm{ml})$ not only disrupted tooth structures, but also promoted expression of osteocalcin (Fig. 2C). Conversely, expression of the important odontogenic marker dentin sialophosphoprotein (DSPP) was suppressed significantly after osteoprotegerin treatment (P<0.001; Fig. 2D-a).

Alteration in osteoprotegerin expression and tooth development after removal of the dental epithelium. The dental mesenchyme was isolated from E14.5 tooth germs and cultured in vitro for 7 days to study whether removal of the dental epithelium increased osteoprotegerin expression and generated a similar effect on tooth development to that observed with extraneous osteoprotegerin. Staining with hematoxylin and eosin revealed that removal of the dental epithelium disrupted tooth structures (Fig. 3A-a and b). RT-qPCR results revealed a significant decrease in expression of the odontogenic marker DSPP in samples of isolated dental mesenchyme
$(\mathrm{P}<0.001$, Fig. 3A-c). Compared with that in tooth germs, expression of osteoprotegerin was significantly upregulated in samples of isolated dental mesenchyme $(\mathrm{P}<0.001$; Fig. 3A-d $)$, as was that of osteocalcin $(\mathrm{P}<0.001$; Fig. 3A-e), RUNX2 $(\mathrm{P}<0.001$; Fig. 3A-f $)$ and ALP $(\mathrm{P}<0.001$; Fig. 3A-g). These results were consistent with data from protein microarrays and the effects of external osteoprotegerin supplementation.

To further confirm these in vitro findings, and to study the effect of removal of the dental epithelium over a long period of time, subrenal capsule cultures were carried out (Fig. 3B). After culture for 3 weeks, implanted tooth germs formed normal tooth structures (Fig. 3C-a1 and a2), whereas implanted mesenchymal tissue formed only irregular structures (Fig. 3C-b1 and b2). Staining with Masson's trichrome revealed that implanted tooth germs formed bone structures and dentin (Fig. 3C-c1 and c2), whereas implanted mesenchyme formed only bone-like structures (Fig. 3C-d1 and d2). Comparison of IHC staining between these two types of implanted tissue revealed that DSP expression was higher in tooth germ-derived tissue (Fig. 3D-b1 and b2). With regard to osteoprotegerin (Fig. 3D-c1 and c2) and 

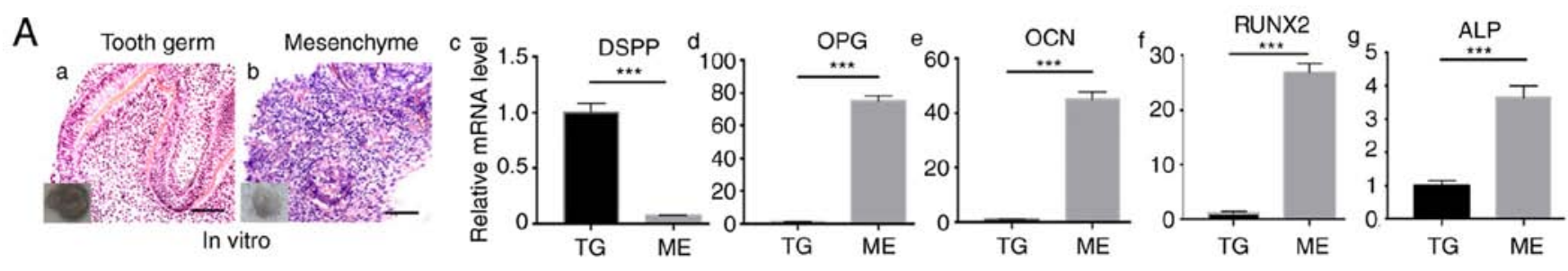

B

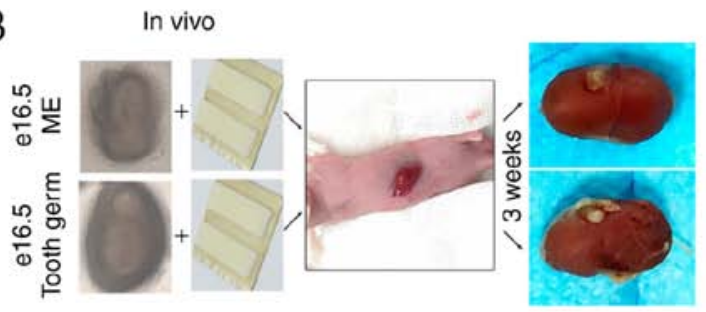

C
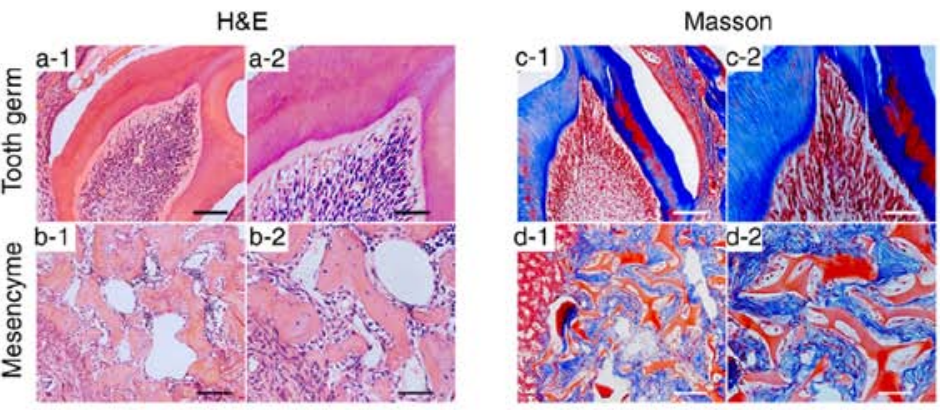

D

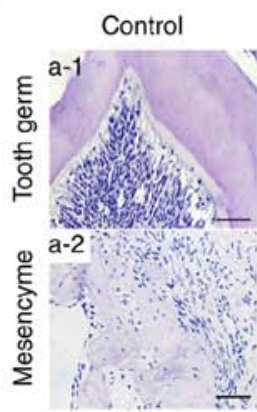

DSP

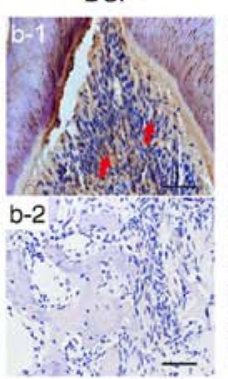

OPG

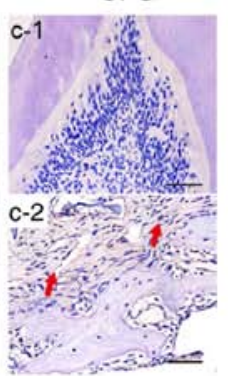

OCN

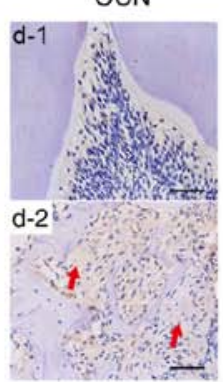

E

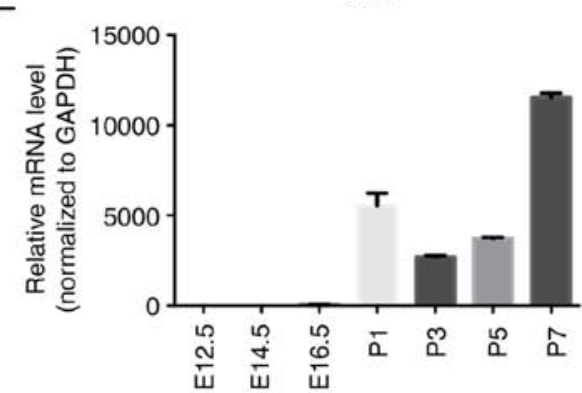

Figure 3. Effects of the removal of the dental epithelium on dental mesenchyme evaluated by in vitro and in vivo cultures. (A) H\&E staining of tooth germs (a) and dental the mesenchyme (b) cultured for 7 days. RT-qPCR revealed that the level of DSPP (c) decreased in the dental mesenchyme, whereas that of OPG (d), OCN (e), RUNX2 (f) and ALP (g) in the dental mesenchyme was significantly higher than that in tooth germs. Each experiment was performed in triplicate. (B) In vivo subrenal capsule culture with gel sponges (schematic). Three E16.5 tooth germs and three samples of the dental mesenchyme were cultured for further study. (C) H\&E staining (a and b) and Masson's trichrome staining (c and d) revealed that, while tooth germs could form normal tooth structures after 3 weeks of subrenal capsule culture, without the dental epithelium, the dental mesenchyme could only form bone-like tissue. (D) (a) Samples without primary antibody were used as negative control. Immunohistochemical staining for DSP (b), OPG (c) and OCN (d) revealed that, after 3 weeks in vivo culture, the DSP level was higher in tooth germ-derived tissue (b1), whereas levels for OPG and OCN were higher in dental mesenchyme-derived tissue (c2 and d2). (E) RT-qPCR revealed that, compared with the prenatal period, OPG expression in postnatal first-molar tooth germs significantly increased. Scale bars, $100 \mu \mathrm{m}(\mathrm{A}-\mathrm{a}$ and $\mathrm{b}, \mathrm{C}-\mathrm{a} 1-\mathrm{d} 1)$ and $50 \mu \mathrm{m}(\mathrm{C}-\mathrm{a} 2-\mathrm{d} 2$ and $\mathrm{D})$. Data are presented as the mean $\pm \mathrm{SD}$. ${ }^{* * * *} \mathrm{P}<0.001$. The red arrows indicate parts of the tissue that were positive for immunohistochemical staining. DSPP, dentin sialophosphoprotein; OPG, osteoprotegerin; OCN, osteocalcin; RUNX2, runt-related transcription factor 2; ALP, alkaline phosphatase; DSP, dentin sialoprotein.

osteocalcin (Fig. 3D-d1 and d2) expression, however, no prominent differences were revealed between the two groups.

As revealed by IHC staining (Fig. 1C), osteoprotegerin expression in the dental mesenchyme increased during postnatal tooth development. RT-qPCR revealed that osteoprotegerin expression in tooth germs increased $>1,000$-fold in the postnatal period (Fig. 3E). These results indicated that suppression by the dental epithelium of osteoprotegerin expression in the dental mesenchyme was relieved in the postnatal period.

Effect of osteoprotegerin on the Wnt/ $\beta$-catenin signaling pathway. The canonical $\mathrm{Wnt} / \beta$-catenin signaling pathway plays an important part in regulation of osteogenic differentiation (16). Hence, the influence of osteoprotegerin on several key molecules in the canonical $\mathrm{Wnt} / \beta$-catenin signaling pathway was investigated (Fig. 4A). mRNA expression of WNT3A was upregulated in whole tooth germs treated with osteoprotegerin ( $\mathrm{P}<0.001$; Fig. 4B-a), whereas that of Dickkopf-related protein-1 (DKK-1, an antagonist of this pathway) was downregulated
$(\mathrm{P}<0.01$; Fig. 4B-b). $\beta$-catenin expression was also significantly increased in osteoprotegerin-treated groups $(\mathrm{P}<0.001$; Fig. 4B-c). Then, nuclear translocation of $\beta$-catenin was evaluated with immunofluorescence assays (Fig. 4C). Compared with the control group, more overlaps between $\beta$-catenin and DAPI were observed in osteoprotegerin-treated tooth germs (especially $200 \mathrm{ng} / \mathrm{ml}$ ) (Fig. 4C-b3 and b4; C-c3 and c4). These results indicated that osteoprotegerin stimulation could enhance canonical Wnt/ $\beta$-catenin signaling.

\section{Discussion}

Removal of the dental epithelium abolishes the normal development of teeth $(17,18)$. In the present study, protein microarray analysis revealed that, after removal of the dental epithelium, osteoprotegerin expression in the dental mesenchyme significantly increased. Järvinen et al (13) revealed that, during prenatal tooth development, the Wnt/ $\beta$-catenin signaling pathway in the epithelium inhibits $\mathrm{Wnt} / \beta$-catenin 

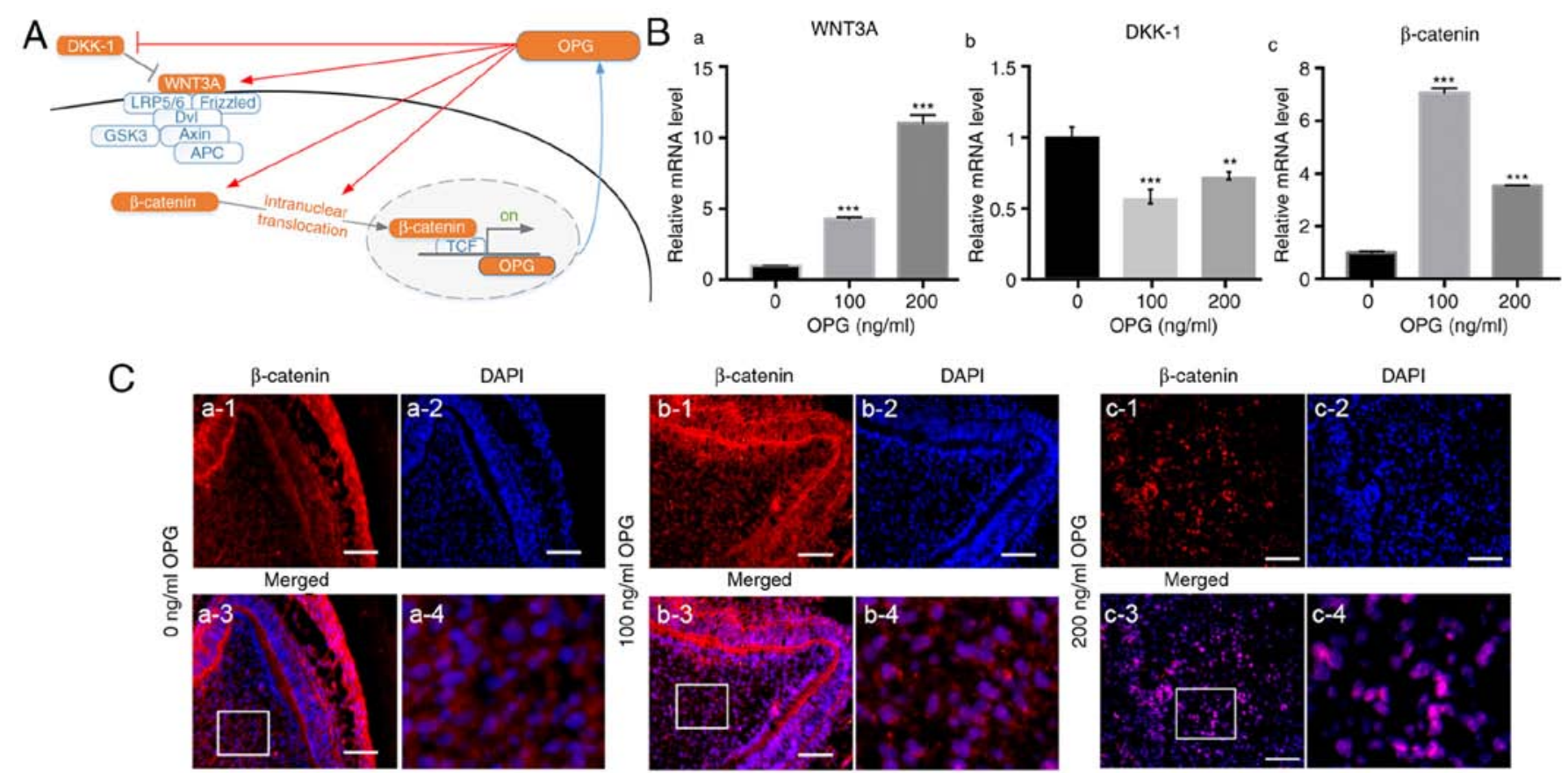

Figure 4. OPG promotes the Wnt/ $\beta$-catenin signaling pathway. (A) Effect of OPG on the Wnt/ $\beta$-catenin signaling pathway, revealed in red arrows (schematic). (B) RT-qPCR revealing that OPG increased the expression of WNT3A (a) and $\beta$-catenin (c) and decreased DKK-1 expression (b) in E14.5 tooth germs. (C) Immunofluorescence assay for intranuclear translocation of $\beta$-catenin. (a) Tooth germ cultured without OPG supplementation was used as control, OPG at 100 (b) and $200 \mathrm{ng} / \mathrm{ml}$ (c) promoted the intranuclear translocation of $\beta$-catenin. Scale bar, $50 \mu \mathrm{m}$ (a1-a3, b1-b3 and c1-c3); a4, b4 and c4 are regional enlargements of a3, b3 and c3. Data are expressed as the mean $\pm \mathrm{SD} .{ }^{* *} \mathrm{P}<0.01,{ }^{* * *} \mathrm{P}<0.001$. OPG, osteoprotegerin; Wnt, wingless/integrated.

signaling in the mesenchyme. Osteoprotegerin expression is promoted mainly by the $\mathrm{Wnt} / \beta$-catenin pathway, which recruits hepatocyte nuclear factor 1 homeobox A (HNF1A, also known as TCF1) to its promoter (19). Hence, removal of the dental epithelium eases suppression of Wnt/ $\beta$-catenin signaling in the dental mesenchyme, leading to upregulation of osteoprotegerin expression. These results indicated that the dental epithelium plays an important role in regulating osteoprotegerin expression in the dental mesenchyme.

In the present study, IHC staining and RT-qPCR revealed that osteoprotegerin expression in the dental mesenchyme was quite low during the prenatal period, but increased significantly after birth. E14.5 tooth germs treated with osteoprotegerin revealed disruption in tooth structures and failure of dentin formation. Further evaluation revealed that osteoprotegerin stimulation promoted expression of the osteogenic biomarkers RUNX2 and osteocalcin, but suppressed DSPP expression, thereby tilting the balance away from odontogenesis. In accordance with our results, Ohazama et al revealed local injection of osteoprotegerin protein or upregulation of osteoprotegerin expression resulted in temporal retardation of tooth development, which may cause defective mineralization in teeth (20). Conversely, a micro-computed tomography study indicated that knockout of osteoprotegerin genes would increase the thickness of enamel and dentin (21). Those studies and our findings indicate that suppression of osteoprotegerin expression in the dental mesenchyme is important for appropriate development of teeth, and that extraneous osteoprotegerin in the early stages of tooth development will disrupt this process.

Results from in vitro cultures revealed that, compared with intact tooth germs, removal of the dental epithelium not only increased osteoprotegerin expression in the isolated dental mesenchyme, but also increased levels of ALP, osteocalcin and RUNX2, and suppressed DSPP expression. Conversely, in vivo cultures revealed no difference in osteoprotegerin expression between tissue derived from tooth germs and isolated dental mesenchyme. Such seemingly inconsistent results could be reconciled by the difference in culture times. For in vitro experiments, tooth germs were cultured for 7 days and, according to a study by Ahmad and Ruch (22), cultured tooth germs develop only to the stage between E16.5 and P1. For in vivo culture, tooth germs or samples of isolated dental mesenchyme were implanted in the subrenal capsule space for 3 weeks. Lungová et al demonstrated that interactions between the dental epithelium and mesenchyme decline gradually after birth (23). Hence, halfway through the 3-week in vivo culture, suppression of osteoprotegerin expression from the dental epithelium had already been removed. Thus, no significant difference in osteoprotegerin expression was revealed between tissue derived from tooth germs and that from samples of the isolated dental mesenchyme.

The Wnt/ $\beta$-catenin signaling pathway plays a critical part in regulation of tooth development $(24,25)$. In the present study, it was revealed that osteoprotegerin was not only a downstream target of the $\mathrm{Wnt} / \beta$-catenin signaling pathway (19), but could also influence Wnt/ $\beta$-catenin signaling. Osteoprotegerin increased WNT3A expression in E14.5 tooth germs, and suppressed DKK-1 expression. In addition, extraneous osteoprotegerin increased expression and intra-nuclear translocation of $\beta$-catenin. Collectively, these results revealed that osteoprotegerin treatment promoted the Wnt/ $\beta$-catenin pathway in E14.5 tooth germs. The Wnt/ $\beta$-catenin signaling pathway has been revealed to promote osteoprotegerin expression (19), thus these results 
indicated a reciprocal activating relationship between osteoprotegerin and the $\mathrm{Wnt} / \beta$-catenin pathway. Choi et al demonstrated reciprocal interactions between $\beta$-catenin and osterix in cementogenesis (26), and a complex interplay between $\mathrm{Wnt} / \beta$-catenin and nuclear factor- $\kappa \mathrm{B}$ signaling pathways in the initiation and maintenance of organ development (27). In the present study, for the first time, evidence was provided for a reciprocal activating relationship between osteoprotegerin and the Wnt/ $\beta$-catenin signaling pathway during the early stages of tooth development. Such interplay could lead to uncontrolled over-activation of $\mathrm{Wnt} / \beta$-catenin signaling in the dental mesenchyme and, as pointed out by Aurrekoetxea et al (28), lead to delays/deficiencies in tooth development.

During the early stages of tooth development, osteoprotegerin expression in the dental mesenchyme was suppressed by the dental epithelium. Addition of osteoprotegerin to prenatal tooth germs promoted the expression of osteogenic markers, inhibited the expression of odontogenic markers, and disrupted tooth structures and dentin formation. In vitro experiments confirmed that removal of the dental epithelium promoted osteoprotegerin expression, and generated a similar effect to that observed upon osteoprotegerin treatment. In vivo experiments revealed similar results. The present data also indicated that the underlying mechanism for these effects is related to enhancement of mesenchymal Wnt/ $\beta$-catenin signaling. Such promotion could further increase osteoprotegerin expression, forming a positive feedback loop, and lead to dysregulation of the early stages of tooth development.

\section{Acknowledgements}

Not applicable.

\section{Funding}

The present study was supported by grants from the National Natural Science Foundation of China (grant no. 81800961), Science and Technology Program of Guangdong Province (grant no. 2016B030229003) and Natural Science Foundation of Guangdong Province (grant no. 2018A030313098).

\section{Availability of data and materials}

The datasets used and/or analyzed during the current study are available from the corresponding author on reasonable request.

\section{Authors' contributions}

$\mathrm{XG}, \mathrm{RZ}$ and $\mathrm{LX}$ contributed to conception and design of the study, XG, JZ and LX contributed to data analysis, ST and BC contributed to data acquisition, and BC, RZ and LX critically revised the manuscript.

\section{Ethics approval and consent to participate}

The protocol for animal studies was approved by the Ethics Committee of the Hospital of Stomatology within Sun Yat-sen University (ERC-2013-15; Guangzhou, China).

\section{Patient consent for publication}

Not applicable.

\section{Competing interests}

The authors declare that they have no competing interests.

\section{References}

1. Tucker A and Sharpe P: The cutting-edge of mammalian development; how the embryo makes teeth. Nat Rev Genet 5: 499-508, 2004.

2. Arakaki M, Ishikawa M, Nakamura T, Iwamoto T, Yamada A, Fukumoto E, Saito M, Otsu K, Harada H, Yamada Y and Fukumoto S: Role of epithelial-stem cell interactions during dental cell differentiation. J Biol Chem 287: 10590-10601, 2012.

3. Xie X, Liu C, Zhang H, Jani PH, Lu Y, Wang X, Zhang B and Qin C: Abrogation of epithelial BMP2 and BMP4 causes amelogenesis imperfecta by reducing MMP20 and KLK4 expression. Sci Rep 6: 25364, 2016.

4. Kanyama M, Shimo T, Sugito H, Nagayama M, Kuboki T, Pacifici M and Koyama E: Regulation of CCN2 gene expression and possible roles in developing tooth germs. Arch Oral Biol 58: 1659-1666, 2013.

5. Gritli-Linde A, Bei M, Maas R, Zhang XM, Linde A and McMahon AP: Shh signaling within the dental epithelium is necessary for cell proliferation, growth and polarization. Development 129: 5323-5337, 2002.

6. Wu X, Li Y, Wang F, Hu L, Li Y, Wang J, Zhang C and Wang S: Spatiotemporal expression of wnt $/ \beta$-catenin signaling during morphogenesis and odontogenesis of deciduous molar in miniature pig. Int J Biol Sci 13: 1082-1091, 2017.

7. Suzuki T, Suda N and Ohyama K: Osteoclastogenesis during mouse tooth germ development is mediated by receptor activator of NFKappa-B ligand (RANKL). J Bone Miner Metab 22: 185-191, 2004.

8. Kearns AE, Khosla S and Kostenuik PJ: Receptor activator of nuclear factor kappaB ligand and osteoprotegerin regulation of bone remodeling in health and disease. Endocr Rev 29: 155-192, 2008.

9. Yamaguchi M: RANK/RANKL/OPG during orthodontic tooth movement. Orthod Craniofac Res 12: 113-119, 2009.

10. Babur C, Ozcan G, Cebi DU, Pervane B, Ozdemir B, Yücel A, Biri AA and Babür C: Gingival crevicular fluid levels of osteoprotegerin (OPG) in premenopausal and postmenopausal women with or without chronic periodontitis. J Dent 40: 364-371, 2012.

11. Dorotheou D, Gkantidis N, Karamolegkou M, Kalyvas D, Kiliaridis S and Kitraki E: Tooth eruption: Altered gene expression in the dental follicle of patients with cleidocranial dysplasia. Orthod Craniofac Res 16: 20-27, 2013.

12. Walsh MC and Choi Y: Biology of the RANKL-RANK-OPG system in immunity, bone, and beyond. Front Immunol 5: 511, 2014.

13. Järvinen E, Shimomura-Kuroki J, Balic A, Jussila M and Thesleff I: Mesenchymal Wnt $/ \beta$-catenin signaling limits tooth number. Development 145: 158048, 2018.

14. Livak KJ and Schmittgen TD: Analysis of relative gene expression data using real-time quantitative PCR and the 2(-Delta Delta C(T)) method. Methods 25: 402-408, 2001.

15. Fingert HJ, Treiman A and Pardee AB: Transplantation of human or rodent tumors into cyclosporine-treated mice: A feasible model for studies of tumor biology and chemotherapy. Proc Natl Acad Sci USA 81: 7927-7931, 1984.

16. Li S, Shao J, Zhou Y, Friis T, Yao J, Shi B and Xiao Y: The impact of Wnt signalling and hypoxia on osteogenic and cementogenic differentiation in human periodontal ligament cells. Mol Med Rep 14: 4975-4982, 2016.

17. Saxen L and Thesleff I: Epithelial-mesenchymal interactions in murine organogenesis. Ciba Found Symp 165: 183-193; discussion 193-188, 1992.

18. Puthiyaveetil JS, Kota K, Chakkarayan R, Chakkarayan J and Thodiyil AK: Epithelial-mesenchymal interactions in tooth development and the significant role of growth factors and genes with emphasis on mesenchyme - a review. J Clin Diagn Res 10: ZE05-ZE09, 2016. 
19. Boyce BF, Xing L and Chen D: Osteoprotegerin, the bone protector, is a surprising target for beta-catenin signaling. Cell Metab 2: 344-345, 2005.

20. Ohazama A, Courtney JM and Sharpe PT: Opg, Rank, and Rankl in tooth development: Co-ordination of odontogenesis and osteogenesis. J Dent Res 83: 241-244, 2004.

21. Sheng ZF, Ye W, Wang J, Li CH, Liu JH, Liang QC, Li S, Xu K and Liao EY: OPG knockout mouse teeth display reduced alveolar bone mass and hypermineralization in enamel and dentin. Arch Oral Biol 55: 288-293, 2010.

22. Ahmad N and Ruch J: Comparison of growth and cell proliferation kinetics during mouse molar odontogenesis in vivo and in vitro. Cell Tissue Kinet 20: 319-329, 1987.

23. Lungová V, Radlanski RJ, Tucker AS, Renz H, Míšek I and Matalová E: Tooth-bone morphogenesis during postnatal stages of mouse first molar development. J Anat 218: 699-716, 2011.

24. Liu F and Millar SE: Wnt/beta-catenin signaling in oral tissue development and disease. J Dent Res 89: 318-330, 2010

25. Liu F, Chu EY, Watt B, Zhang Y, Gallant NM, Andl T, Yang SH, Lu MM, Piccolo S, Schmidt-Ullrich R, et al: Wnt/beta-catenin signaling directs multiple stages of tooth morphogenesis. Dev Biol 313: 210-224, 2008.
26. Choi H, Kim TH, Yang S, Lee JC, You HK and Cho ES: A reciprocal interaction between $\beta$-catenin and osterix in cementogenesis. Sci Rep 7: 8160, 2017.

27. Zhang Y, Tomann P, Andl T, Gallant NM, Huelsken J, Jerchow B, Birchmeier W, Paus R, Piccolo S, Mikkola ML, et al: Reciprocal requirements for EDA/EDAR/NF-kappaB and Wnt/beta-catenin signaling pathways in hair follicle induction. Dev Cell 17: 49-61, 2009.

28. Aurrekoetxea M, Lopez J, Garcia P, Ibarretxe G and Unda F: Enhanced Wnt/ $\beta$-catenin signalling during tooth morphogenesis impedes cell differentiation and leads to alterations in the structure and mineralisation of the adult tooth. Biol Cell 104: 603-617, 2012.

This work is licensed under a Creative Commons Attribution-NonCommercial-NoDerivatives 4.0 International (CC BY-NC-ND 4.0) License. 